Sigurd Paul Scheichl ${ }^{*}$

Universität Innsbruck
UDK 821.112.2(100).09:028(430+436)

DOI: 10.4312/linguistica.60.2.283-297

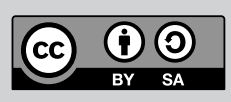

\title{
DAS ,ZENTRUM“ UND DIE LITERATUR VON DEN „RÄNDERN“. DIE REZEPTION DER LITERATUR DER DEUTSCHEN MINDERHEITEN IN DEUTSCHLAND UND ÖSTERREICH
}

Trotz manchen Vorbehalten ist für unser Thema Karl Kurt Kleins Literaturgeschichte des Deutschtums im Ausland (Klein 1939) nach wie vor unumgänglich; neben den entsprechenden, allein Regionen Österreich-Ungarns behandelnden Abschnitten in der 1937 abgeschlossenen Deutsch-österreichischen Literaturgeschichte von Nagl, Zeidler und Castle (Nagl/Zeidler/Castle 1899 bis 1937) nennt allein sie eine Fülle von Namen, die man außerhalb der Herkunftsregionen nicht kennt/kannte - und vielleicht nicht einmal dort. Die von diesem Handbuch gebotenen Informationen werde ich nicht ergänzen, ich werde nur auf seiner Grundlage einige Fragen aufwerfen, die es nicht oder nur in Ansätzen stellt.

Zuerst müssen aber zwei Vorbehalte gegen Klein ausgesprochen werden: Eine kleine, aber in diesem Rahmen jedenfalls kritisch anzumerkende Lücke ist das Fehlen der Autoren aus den deutschsprachigen Inseln im einstigen Herzogtum Krain und in den überwiegend slowenischen Teilen der einstigen Steiermark. Die Steiermark war für Klein eben deutsch, wie auch die Prager Autoren für ihn keine „Auslanddeutschen“ waren. Dass sie wie beispielsweise Anastasius Grün nicht als Sprachinseldichter wahrgenommen werden und daher in diesem Werk fehlen, ist unter anderem mit der zumal in den städtischen Zentren noch unbestrittenen Stellung des Deutschen in der ersten Hälfte des 19. Jahrhunderts zu erklären.

Viel gewichtiger ist Kleins Trennung von „deutschsprachigen“ und „echt volksdeutschen“ Literaten und Literatinnen. Ein heute peinliches Zitat zu diesem Problem:

Vielfach war die damals [vor und kurz nach 1900] in Ungarn entstehende deutschsprachige Literatur in Wirklichkeit jüdisches Schrifttum in deutschem Gewande. Rein geistig und künstlerisch betrachtet stand es oft hoch und wurde, ähnlich wie Sacher-Masoch in Galizien oder Karl Emil Franzos in der Bukowina, von den Führern der öffentlichen Meinung hoch genug eingeschätzt, um über Wien, Österreich und das Deutsche Reich in die Weltliteratur eindringen zu können (was echtem volksdeutschen Schrifttum aus diesen Gebieten nie widerfahren ist). (Klein 1939: 228)

sigurd.p.scheichl@uibk.ac.at 
(Warum wohl?) Wieweit solche Formulierungen mit den politischen Bedingungen der Entstehungszeit zu erklären oder Zuspitzungen von Kleins Denken sind, in dem der altösterreichische Deutschnationalismus seine Spuren hinterlassen hat, lasse ich offen; eine Auseinandersetzung mit seinem Werk ist hier nicht meine Aufgabe. Die Folgen dieser Auffassung möchte ich am Beispiel der Bukowina zugespitzt vorführen: Deren blühende Literatur aus den dreißiger Jahren würde sich auf - den echten Volksdeutschen Georg Drozdowski reduzieren.

Trotz diesen - und anderen - grundsätzlichen Einwänden beginne ich mit zwei Zitaten aus Kleins monumentalem Werk: „Es ist kein Fall bekannt, dass außerdeutsches Schrifttum als solches vor dem Kriege dem Binnendeutschtum näher bekannt gewesen wäre.“ (Klein 1939: 266) Und: „Kein großer, aber ein siebenbürgisch-sächsischer Dichter zu sein - dies zur Kennzeichnung Michael Alberts geprägte Wort [...]" (Klein 1939: 241) Das erste Zitat widerlegt Klein übrigens selbst in den Abschnitten über die schriftstellerischen Leistungen des „Herrenvolks“ der Baltendeutschen, die von Lenz bis Bergengruen im gesamten Sprachraum wahrgenommen worden sind (Klein 1939: 167), wozu die geografische Nähe zu Königsberg und selbst zu Berlin beigetragen haben dürfte. (Im Weiteren verzichte ich auf baltendeutsche Beispiele.)

Für die Autoren der südostdeutschen Minderheiten, die mich besonders interessieren und auf denen übrigens emotional der Schwerpunkt von Kleins Kompendium liegt, stimmt der Satz allerdings. Geografisch und noch mehr politisch lag das ohnehin weit genug entfernte Wien am nächsten - aber die Hauptstadt der Donaumonarchie war trotz ihren bedeutenden Autoren kein Zentrum des literarischen Lebens und vor allem keines des Literaturbetriebs. Das ist ein Grund für die geringe Rezeption zumal der südostdeutschen Literaten und Literatinnen im „binnendeutschen“ Raum, doch nicht der einzige und wohl nicht einmal der wichtigste.

Die beiden Klein-Zitate verweisen auf zwei Probleme der Rezeption von deutschen Sprachinselautoren. Die Einschätzung Michael Alberts wirft die Frage auf, wieweit Autoren aus Siebenbürgen, dem Banat oder einer anderen Sprachinsel ihre Aufgabe nicht in erster Linie darin sahen, für ihre engeren Landsleute zu wirken und deren kulturelles Selbstbewusstsein zu stärken, selbst durch Beiträge zu den lokalen Kalendern. Das geschah auch durch die Pflege der Mundart ${ }^{1}$; die Zahl der Dialektdichter und -dichterinnen ist unter den Auslanddeutschen nicht gering. Da sie sich von vornherein nur an die engsten Landsleute wenden und eine Barriere für Leser und Leserinnen außerhalb ihrer Region aufgebaut haben, werde ich auf sie im Weiteren gar nicht eingehen.

Aber auch Autoren und Autorinnen, die sich der Standardsprache bedienten, blieben oft durch lokale Themen und Motive bewusst der Region ihrer Herkunft so stark verbunden, dass sie außerhalb derselben nicht wahrgenommen wurden und auch nur schwer wahrgenommen werden konnten; das umso weniger, je unbekannter die Lebensbedingungen der jeweiligen Sprachinsel dem Publikum in Österreich und noch mehr in Deutschland waren. Diese regionale Bindung hatte auch ihre Vorzüge: Denn man hatte

1 Eine schöne Stelle über die Selbstverständlichkeit der (Banater) Mundart und ihre mögliche oder nicht mögliche Verwendung im standardsprachlichen Roman findet sich bei Lippet (Lippet 2015: 12f.). 
zwar nur wenige Leser und Leserinnen, aber man konnte sich deren Wohlwollens und der Zustimmung der regionalen literarischen Autoritäten sicher sein. Obendrein konnte man in der Region traditioneller schreiben und brauchte sich nicht den aus den großen literarischen Zentren kommenden Neuerungen anschließen. Kleins gelegentliches Lob, jemand habe sich dem Expressionismus entzogen (Klein 1939: 379, 393, 421), ist in dieser Hinsicht aufschlussreich (und gewiss nicht nur mit den politischen Bedingungen der Entstehungszeit zu erklären).

Man muss also fragen, ob ein Autor beispielsweise aus Galizien überhaupt die Absicht hatte, aus der Regionalliteratur auszutreten ${ }^{2}$, ob er sich nicht vor allem an (deutschsprachige) Leser und Leserinnen in Galizien wenden wollte und nur beschränkt Interesse an einem breiteren Publikum hatte, ob er mutig genug war, das Risiko einzugehen sich einer nicht unbedingt wohlwollenden Kritik in den literarischen Zentren auszusetzen, nicht zuletzt, ob sein Werk gut genug war, um vor der Leserschaft des gesamten Sprachraums zu bestehen. Verschärft wurde das Dilemma dadurch, dass die deutschen Minderheiten seit der Mitte des 19. Jahrhunderts fast überall durch ihr anderssprachiges Umfeld bedroht waren, so dass ein Autor es sehr wohl als seine Hauptaufgabe ansehen konnte, seine engsten Landsleute durch sein Werk zu unterstützen, auch wenn er durch diese Thematik außerhalb seiner Region unverständlich oder doch uninteressant zu werden drohte.

Da wir von den meisten in Frage kommenden Autoren relativ wenig wissen, wird über die Absicht, aus der Regionalliteratur auszutreten, nur selten etwas zu ermitteln sein; allenfalls kann man aus der Wahl des Verlags Schlüsse ziehen: Aber auch da wissen wir ja zumeist nicht, ob und wie viel Ablehnungen durch binnendeutsche Verlage dem Druck durch Krafft und Drotleff in Hermannstadt vorausgegangen sind. Traugott Teutsch (1829-1913) jedenfalls hat davon berichtet, wie er in Berlin gescheitert ist, als er, in Siebenbürgen durch mehrere Bücher bereits bekannt, ein reichsdeutsches Familienblatt für ein Romanmanuskript von ihm zu interessieren versucht hat (Sienerth 2008b: 48f.). ${ }^{3}$ (Um Missverständnisse zu vermeiden, sei gesagt, dass das Problem der Wirkung außerhalb der Heimatregion selbstverständlich alle Autoren und Autorinnen außerhalb der Zentren betrifft, Steirer wie Pfälzer und Oldenburger, keineswegs nur die Literaten und Literatinnen aus den Sprachinseln; bei ihnen mag es freilich durch die geografischen Bedingungen verschärft sein.)

Für Bemühungen der Autoren selbst um Rezeption außerhalb der Region bietet Alfred Margul-Sperber (1898-1967) ein Beispiel. Im Nachlass Ludwig v. Fickers, des Herausgebers des Brenner, liegt ein Exemplar von Margul-Sperbers Gleichnissen der Landschaft (Storoshinez 1934, also wohl im Selbstverlag), mit einer Visitenkarte des Autors, auf deren Rückseite gedruckt steht: „Überreicht vom Verfasser mit der Bitte um gefl. briefliche Äußerung eines Urteils.“4 - nicht einmal um eine Besprechung.

2 Grundsätzlich zu diesem Problem Sienerth (2008: 37-55). Dazu ferner Scheichl (1993: 33-49).

3 Allerdings sind 1891 Werke von Teutsch und von Michael Albert in der Zeitschrift Das 20. Jahrhundert erschienen (Sienerth 2008: 52).

4 Brenner-Archiv, Universität Innsbruck, Bibliothek IV-188. 
Briefe von Margul-Sperber an Ficker oder Entwürfe Fickers zu Antworten befinden sich im Brenner-Archiv allerdings nicht. Margul-Sperbers Kontakte mit Karl Kraus und mit Josef Weinheber deuten ebenfalls darauf hin, dass er bereit war, sich dem Urteil der literarischen Zentren zu stellen, und über die Bukowina hinaus wirken wollte.

Leichter kommt man dem Problem bei, wenn man von den Autoren ausgeht, die tatsächlich im gesamten Sprachraum erfolgreich gewesen sind. Der erste Dichter aus den uns hier interessierenden Regionen, auf den das zutrifft, ist Nikolaus Lenau (18021850 ) aus dem Banat. Seine Erfahrungen weniger im Banat als im rein madjarischen Gebiet von Tokaj, in dem er wichtige Jahre seiner Jugend verbrachte, bestimmen den Motivschatz vor allem seiner Lyrik. Aber: Als seine Gedichte bei Cotta in Stuttgart erschienen, lebte er bereits in Württemberg (und vorher schon in Wien).

Die Integration eines Autors in das binnendeutsche literarische Geschehen hängt nicht nur bei Lenau mit seinem Wohnort zusammen. Anastasius Grün (Anton Alexander von Auersperg, 1806-1876) ist in Ljubljana/Laibach geboren und hat mit Übersetzungen slowenischer Volkslieder (Volkslieder aus Krain, Leipzig: Weidmann 1850) seine Herkunft in sein literarisches Werk einbezogen, wechselte Briefe mit dem klassischen slowenischen Dichter Prešeren, hat auch immer wieder auf seinen Gütern im damaligen Krain gelebt und war in die Politik dieses Kronlands eingebunden, aber entscheidend für seine ,überregionale“ literarische Präsenz war das, was er in und über Wien geschrieben hat, wo er einen großen Teil seines Lebens verbrachte. (Grün ist übrigens, sieht man von einigen Autoren aus Prag ab, einer der ganz wenigen deutschsprachigen österreichischen Autoren, der eine in der Monarchie gesprochene slawische Sprache zumindest halbwegs beherrscht hat.)

Von „Binnenmigration“ innerhalb der Habsburgermonarchie könnte man bei einer ganzen Reihe von Autoren und Autorinnen sprechen, deren Platz in der Literaturgeschichte wahrscheinlich ein anderer wäre, wenn sie in ihrer Sprachinsel geblieben oder nach dem Studium auf Dauer in sie zurückgekehrt wären. Karl Emil Franzos (18481904), der nach vielen Jahren in Wien noch nach Berlin weiter gezogen ist, wäre ein weiteres Beispiel. Das gilt noch mehr für den neben Lenau fast einzigen Autor aus den Sprachinseln, der zweifellos zur Weltliteratur gehört: für Joseph Roth; selbstverständlich fehlt er bei Klein (was dem Autor wohl recht gewesen wäre). Roth hat Galizien im Alter von etwa 20 Jahren auf Dauer verlassen und ist zum Wiener und Berliner Schriftsteller geworden; freilich hat er vor allem nach 1930 immer wieder galizisch-jüdische Motive aufgegriffen (während ihn die „Volksdeutschen“ seiner Heimatregion nur wenig interessiert haben dürften).

Einige andere Fälle, bei denen das Verlassen der Region den Austritt aus der Regionalliteratur bewirkt oder erleichtert hat, kommen bei Klein vor, jedoch nicht viele. Marie Eugenie delle Grazie (1864-1931) ist im Banat geboren, kam aber schon als Kind nach Wien, wo sie ,eine große Gemeinde um sich“ sammeln sollte. Bezüge des Werks zu ihrer Heimat seien selten (Klein 1939: 251). Andererseits war es ihr im Wiener Umfeld möglich zeitgemäßen Verfahrensweisen zu folgen, in ihrem Fall denen des Naturalismus (Millner 2016), was ihr, wenn sie im Banat geschrieben hätte, nicht so leicht möglich gewesen wäre, weniger wegen mangelnder Informationen als wegen des 
hauptstadtfernen Publikums. Klein rügt geradezu, dass selbst die Dorfgeschichten der Autorin nicht frei von Spuren des Naturalismus seien (Klein 1939: 251).

Einer späteren Generation gehört der erfolgreiche Franz Xaver Kappus (18831966), ebenfalls aus dem Banat, an. Er hat immer wieder südosteuropäische Motive verwendet, doch kommt es ihm weniger auf diese als auf die unterhaltende Wirkung seiner Prosa an. Den Erfolg als Unterhaltungsautor dankt Kappus, bis 1925 Journalist in Temeswar, gewiss auch dem Entschluss, das Banat zu verlassen und nach Berlin zu gehen, wo Kontakte zu den entsprechenden Verlagen leicht herzustellen waren (vgl. Klein 1939: 406f., mit zum Teil recht bedenklichen Formulierungen).

Ein wichtiger Sonderfall ist Adam Müller-Guttenbrunn (1852-1923), dessen späte historischen Romane $e^{5}$ überregional erfolgreich gewesen sind, vor allem bei tendenziell deutschnationalen und an den deutschen Minderheiten ohnehin interessierten Lesern und Leserinnen. Klein hält zu Recht fest: „Für die Sendung, die [Müller-Guttenbrunn] erfüllen sollte, ist es ein besonderer Glücksfall gewesen, dass sich der Dichter als Gereifter und Vollendeter der Gestaltung seiner donauschwäbischen Heimatstoffe widmete." (Klein 1939: 256) Man kann es schlichter ausdrücken: Der Autor, der das Banat schon in jungen Jahren verlassen hatte, war bereits eine prominente Figur im literarischen Leben Wiens, als er sich ungarndeutschen Themen zuwandte; er war schon längst aus der Regionalliteratur ausgetreten, hatte ihr eigentlich nie angehört, als er donauschwäbische Stoffe zu behandeln begann. „Der Heimatdichter Adam Müller-Guttenbrunn“ „erstand““ erst mit dem Roman Götzendämmerung von 1907 (Klein 1939: 257) - als der Autor bereits 55 Jahre alt war. Für uns ist unabhängig von der politischen Wirkung seiner Romane bei den Donauschwaben und im binnendeutschen Raum wichtig, dass der Erfolg seiner späten Bücher außerhalb des Banats der Erfolg eines Autors war, der zur Wiener Literaturszene gehörte und nie zu der von Temeswar gehört hatte. Somit bestätigt auch Müller-Guttenbrunn meine Hypothese, dass der Austritt aus der Regionalliteratur zumeist als geografischer Austritt funktioniert hat (und wohl auch noch funktioniert) - wobei die Veränderung des Wohnorts eine Fülle von Folgen hatte, insbesondere das Knüpfen neuer Beziehungen sowohl zur binnendeutschen Literaturszene als auch zu den großen Verlagen ermöglicht. Das größere und besser informierte Publikum wirkt auch auf das Werk zurück. (Die Übersiedlung in die Nähe der literarischen Zentren ist freilich keine Garantie für den Erfolg, was etwa die Nicht-Karriere des nach Wien übersiedelten Kronstädters Egon Hajek, 1888-1963, zeigt.)

Auch der so stark in Siebenbürgen verankerte Heinrich Zillich (1898-1988), der sich durch die Leitung der Zeitschrift Klingsor (ab 1924) große Verdienste um die Öffnung der siebenbürgischen Literaturszene erworben hatte, hat schließlich seine Heimat verlassen und ist, unter den besonderen Bedingungen des NS-Regimes, 1936 ins Deutsche Reich übersiedelt. Das mag zu seinen zeitweiligen literarischen Erfolgen beigetragen haben.

Eine andere Möglichkeit, die regionalen Grenzen zu überschreiten, hat es vor allem in Ungarn gegeben: den Wechsel der Sprache. „Der schwäbische Schriftsteller Franz

5 Zu Joseph dem Deutschen (1917) vgl. jetzt Jachimowicz (2018: 205-221). 
Herzog, dessen dichterische Anfangsversuche noch deutsch waren, wurde als Herczeg Ferenc [1863-1954] der führende ungarische Erzähler der Gegenwart [...]“ (Klein 1939: 250). Vor allem in der Zips scheint dieser Weg nicht selten gegangen worden zu sein, bis hin zu Márai Sándor (1900-1998).

Der Austritt aus der Regionalliteratur durch die Übersiedlung nach Wien oder Berlin bestimmt auch die Erfolge von Autoren aus den Sprachinseln nach 1945. Karl Dedecius (1921-2016), der in Łódź aufgewachsene große Vermittler der polnischen Literatur, wäre ein Beispiel für solches räumliches und geistiges Verlassen der Region, das in seinem Fall freilich erzwungen war.

Georg Maurer (1907-1971), bei Klein schon mit sechs Zeilen und einem Zitat als Gedankenlyriker erwähnt (Klein 1939: 428), ist 1926 nach Deutschland gekommen und dort geblieben; in der Deutschen Demokratischen Republik gehörte er zu den angesehenen Autoren und fand sicher mehr Leser, als er in Siebenbürgen (wo von ihm Gedichte im Klingsor erschienen sind) gefunden hätte.

In der Deutschen Demokratischen Republik, erschienen auch Alfred Margul-Sperber (Margul-Sperber 1973) und der erste Gedichtband von dem damals noch in Klausenburg lebenden Franz Hodjak (* 1944) außerhalb Rumäniens (Hodjak 1988) ${ }^{6}$, eine Auswahl aus mehreren in Rumänien gedruckten Gedichtsammlungen. 1990 folgte bei Suhrkamp eine Auswahl von Gedichten Hodjaks, der durch seine Teilnahme am Bachmann-Wettbewerb von 1990 und seine Auswanderung in die Bundesrepublik (1992) zu einer bekannten literarischen Figur wurde. Insgesamt spielte die Deutsche Demokratische Republik zwar bei der Vermittlung ungarischer, rumänischer, bulgarischer usw. Literatur ins Deutsche eine verdienstvolle Rolle; die auslands-, $d$. h. für damals fast nur noch die rumäniendeutschen Autoren und Autorinnen haben die Verlage in Ost-Berlin weniger interessiert

Was für Hodjak gilt, trifft - ohne ein Buch in der DDR - ähnlich auf die zum Teil lange vor ihm in die Bundesrepublik gekommenen Autoren und Autorinnen aus dem Banat und aus Siebenbürgen zu: Oskar Pastior, Richard Wagner, Johannes Lippet, Herta Müller u. v. a. Im gesamten Sprachraum als Literaten und Literatinnen wahr- - und dann zu Recht sehr ernst - genommen sind sie durchwegs erst nach dem Verlassen Rumäniens worden; vorher kannte man sie allenfalls als politisch Verfolgte und in kleinen Kreisen. Dass in ihren Büchern spezifisch rumänische Erfahrungen gestaltet sind, hat ihrer Rezeption nicht geschadet. Der in vielen Fällen gelungene räumliche Austritt aus der Regionalliteratur ist anders als in den Jahren vor 1945 nur in seltenen Fällen ganz freiwillig gewesen.

Ein einziger Autor aus Siebenbürgen ist in Österreich von einem großen Verlag veröffentlicht, aber auch in Deutschland sehr intensiv rezipiert worden (vgl. Scheichl 2019), ohne dass er seine Heimat verlassen hätte: Eginald Schlattner (*1933). Das hat mit der Qualität seines Erzählens zu tun, aber auch damit dass das Ende der siebenbürgisch-sächsischen Geschichte um 1990 vielen Deutschen und Österreichern bewusst

6 Schon 1987 war in der DDR-Reihe „Poesiealbum“ ein schmales Heft mit Versen von Hodjak erschienen. 
war und sie Schlattner als den Chronisten dieser Ereignisse lasen. Schlattners frühe Erzählungen sind nach dem Erfolg seiner Romane aber doch in Hermannstadt erschienen (Schlattner 2012), sein vierter Roman, Wasserzeichen, zwar in der Bundesrepublik, aber in dem kleinen, auf rumäniendeutsche und rumänische Literatur spezialisierten POP-Verlag (Schlattner 2018).

Fast die einzige ältere Ausnahme ist Otto Alscher (1880-1944) (zu ihm vgl. Klein 1939: 404f.), der früh in das heimatliche Banat zurückgekehrt und dort geblieben ist. Seine Zigeuner- und Tiergeschichten sind durchwegs in größeren, für Neues offenen binnendeutschen Verlagen erschienen; auch der Innsbrucker Brenner enthält Beiträge von ihm. Dieser Erfolg außerhalb der Heimatregion lässt sich außer mit der Qualität seiner Novellen und Romane vielleicht auch damit erklären, dass er in seiner Jugend zur Wiener Boheme gehörte und später als zeitweiliger Mitarbeiter der angesehenen, auch außerhalb Ungarns gelesenen Zeitung Pester Lloyd nützliche Kontakte zur Literaturszene hat knüpfen können. (Am Rande: Gar nicht wenige Neuauflagen von Büchern Alschers sind nach 1945 erschienen, fast ausschließlich in Bukarest.)

Eine interessante Parallele zum Zusammenspiel von Wohnort und Rezeption bieten die erfolgreichen englischsprachigen Autoren und Autorinnen aus dem Commonwealth, die zu einem guten Teil die Karibik oder Indien verlassen haben und nach Großbritannien oder in die USA übersiedelt sind, wie etwa der lange in Boston tätige Derek Walcott oder der heute vor allem in New York lebende Salman Rushdie. ${ }^{7}$

So viel zur Absicht von Autoren und Autorinnen, außerhalb der Sprachinsel-Region wahrgenommen zu werden, und den Schritten, die sie in diesem Sinn unternommen haben - bis zum Verlassen der Heimat. Dabei gehe ich weder auf die vielen Fälle ein, in denen Schriftsteller und Schriftstellerinnen vielleicht versucht haben, außerhalb ihrer Sprachinsel Leser und Leserinnen zu finden, ohne mit ihrem Bemühen Erfolg gehabt zu haben, noch auf jene, gewiss zahlreichen, Personen, deren Absicht es im Sinn der einleitend zitierten Charakterisierung Michael Alberts gewesen ist, einen Beitrag zur kulturellen Identität ihrer Heimat zu leisten, nicht weniger, aber eben auch nicht mehr. Die weit überwiegende Zahl der von Klein und in Nagl/Zeidler/Castle vorgestellten Personen waren ja alles eher als Berufsschriftsteller. Viele waren Lehrer und Pfarrer und hatten schon aus ihrem sozialen Umfeld heraus keinen besonderen literarischen Ehrgeiz.

Gadeanu (2011) versucht am Beispiel des Banats das Verhältnis von Sprachinselautoren und -autorinnen zur Literatur des Zentrums mit dem Konzept der „,kleinen Literatur" von Deleuze und Guattari zu präzisieren. An diesem Ansatz zweifle ich grundsätzlich, weil ihn die beiden Autoren aufgrund einer gelegentlichen Bemerkung Kafkas (über die jiddische Literatur in Polen und über die wieder aufblühende tschechische Literatur) ohne empirische Grundlage und ohne nähere Kenntnis der literarischen Situation im östlichen Mitteleuropa entwickelt haben. Zumindest das Selbstverständnis der Prager Autoren, die zumeist in reichsdeutschen Verlagen veröffentlichten (auch die weniger bekannten wie etwa Hugo Salus) war alles eher denn das von Angehörigen einer „kleinen Literatur“. Das mag im (zumal nach 1945) vom Binnenraum abgeschnittenen Banat anders gewesen sein. Das Schwanken der Schriftsteller und Schriftstellerinnen

7 Diesen Hinweis verdanke ich Wolfgang Zach. 
aus dem Banat zwischen der Orientierung an einem lokalen (,kleine Literatur“) und einem weiteren Publikum (der ,deutschen Literatur“) zeigt Gadeanu unabhängig von seinem theoretischen Ansatz jedenfalls sehr eindringlich.

Nun zur zweiten Frage, die sich aus dem ersten der beiden Klein-Zitate ergibt, mit denen ich begonnen habe. Es geht darum, wie man in der binnendeutschen Literaturszene mit Texten aus den Sprachinseln umgegangen ist, ob „binnendeutsche“ Verlage (und damit Leser und Leserinnen) an Autorinnen und Autoren aus den Sprachinseln Interesse gehabt haben und haben (vgl. Scheichl 1992). Klein geht auf das Problem übrigens nur selten ein, so wenig wie auf die nach dem die Grenzen der Region überschreitenden Ehrgeiz der „,auslanddeutschen“ Autoren und Autorinnen. Am ehesten klagt er über Desinteresse an diesen, sogar in verfänglich zeittypischen Formulierungen wie: „Den im Reich angesessenen Rassefremden erkannte man als Deutschen an, den Blutsdeutschen der Fremde aber - mit dem kultürlich und geistig allerdings oft wenig Staat zu machen war - nicht.“ (Klein 1939: 263)

Der Rezeption auslanddeutscher Literatur standen auch objektive Schwierigkeiten entgegen. Es war für Verlage im Deutschen Reich und in Österreich schon aus ganz banalen Gründen nicht leicht, Autoren und Autorinnen aus den Sprachinseln zu entdecken. Man hätte nach ihnen fahnden müssen, denn sie waren nicht präsent; ihre Texte standen selten oder nie in den Zeitungen und Zeitschriften, die man las, man traf sie nicht bei kulturellen Veranstaltungen, sie gehörten nicht den Wiener, Münchner oder Berliner literarischen Vereinigungen an. Die Verleger kannten in der Regel niemand, der in Odessa ${ }^{8}$, Hermannstadt oder Temeswar Schriftsteller kennen gelernt und sie in Leipzig empfohlen hätte. Selbst wenn man Interesse an einem Autor aus den fernen Regionen gehabt hätte, war es schwer mit ihm persönlichen Kontakt aufzunehmen; Reisen von Berlin nach Czernowitz oder von Czernowitz nach Berlin waren umständlich. Das gilt wie für die Verleger so auch für die Herausgeber von Zeitschriften, die für die Entdeckung von Autoren noch wichtiger waren als die Verlage. Kaum war ein Autor nach Wien übersiedelt, konnte er den Weg in die diversen Cliquen finden, die den Weg zum literarischen Erfolg öffneten, in Wien in die liberalen wie Franzos oder in die antisemitischen wie Müller-Guttenbrunn.

Ein weiteres Problem war das geringe Wissen über die Sprachinseln sowohl im Deutschen Reich als auch in Österreich-Ungarn, in dem die Frage der sich bedroht fühlenden deutschen Minderheiten erst gegen Ende des Jahrhunderts durch den wachsenden Nationalismus und besonders durch die Schulvereine in größerem Maße bewusst geworden ist. Auch deshalb ist von einem besonderen Interesse der Verlage an auslanddeutschen Autoren und Autorinnen nicht auszugehen, wie sie nicht mit besonderem Interesse des Publikums an Büchern aus diesen Regionen und über deren Probleme rechnen konnten. Ganz unberechtigt sind Kleins wiederholte Klagen über die binnendeutsche Gleichgültigkeit gegenüber den Auslanddeutschen nicht. Nicht zuletzt waren

8 Auch Wilhelm Wolfsohn (1820-1865) aus Odessa, kein Volksdeutscher und daher bei Klein nicht erwähnt, verließ die Ukraine bzw. Russland und wurde dann in Dresden ein wichtiger Vermittler der russischen Literatur. 
auslanddeutsche Werke bis zu einem gewissen Grad kommentarbedürftig, denn welche binnendeutsche Leserin wusste schon Bescheid über ungarische Speisen in den deutschen Siedlungsgebieten Ungarns oder über die Bedingungen, unter denen die deutschen Kolonien in Russland entstanden waren. Schwierigkeiten mit der Sprache der Autoren und Autorinnen aus den Minderheitengebieten gab es dagegen kaum; sie verwendeten die Standardsprache und streuten nur vereinzelt Regionalismen oder Wörter aus den Fremdsprachen ihres Umfelds ein.

Das Hauptproblem für Veröffentlichungen von Sprachinselautoren und -autorinnen im binnendeutschen Raum war schließlich die Qualität der Texte. Selbst Karl Kurt Klein gesteht an einer bereits zitierten Stelle ein, dass mit den „Blutsdeutschen der Fremde“ „,kultürlich und geistig [...] oft wenig Staat zu machen war [...].“ (Klein 1939: 263). Das hatte viele Gründe; einer wird die Abgeschnittenheit vielleicht noch mehr der Leser und Leserinnen als der Autoren und Autorinnen von den geistigen Zentren gewesen sein, ein anderer die gesellschaftlichen Gegebenheiten in den Siedlungsgebieten der Auslanddeutschen: Fast überall dominierten die Bauern, außer in Siebenbürgen war die Bildungsschicht dünn und sie hatte (wiederum mit Ausnahme der Siebenbürger Akademiker) zumeist ihre Ausbildung in fremden Sprachen erfahren. Diesem regionalen Publikum, an das man sich ja doch wenden wollte, war allzu Komplexes nicht zuzumuten, was wiederum dazu führte, dass man sich Neuerungen der Literatur in den Zentren nicht oder nur verspätet anschloss und daher ,altmodisch“ war. Die mangelnde Synchronie der Literatur der „Volks-“ mit jener der „Binnendeutschen“ hat gewiss zum Desinteresse der binnendeutschen Verlage an der Literatur der Auslanddeutschen beigetragen. (Allein bei der rumäniendeutschen Literatur nach 1945 und schon bei den Lyrikern der Bukowina in den 30er Jahren hat es diesen Abstand nicht mehr gegeben.)

Und noch einmal: Das gilt nicht für die Autoren und Autorinnen, die in den binnendeutschen Raum übersiedelt waren. Für die galten keine anderen Kriterien als für jene, die seit jeher in Wien, Graz, München oder Leipzig schrieben. Der sicherlich interessante Karl Emil Franzos gehört nicht zu den Großen des deutschen Realismus, war aber als Realist erfolgreich; Marie Eugenie delle Grazie stand dem Naturalismus nahe; Kappus war ohnehin ein Unterhaltungsautor und nicht mehr; Müller-Guttenbrunn war eher aus politischen denn aus literarischen Gründen populär und selbst begeisterte Zeitgenossen haben wohl nicht damit gerechnet, dass er einmal zum Kanon der deutschen Literatur gehören könnte. Wenn Autoren und Autorinnen aus den Sprachinseln ein Plätzchen in der binnendeutschen Literaturszene gefunden haben, so haben sie das jedenfalls nur selten Initiativen der Verlage aus Wien, Berlin usw. zu danken.

Der Vollständigkeit halber möchte ich doch darauf hinweisen, dass Autoren und Autorinnen aus manchen Sprachgruppen überhaupt nie in Österreich und Deutschland Beachtung gefunden haben: Werke von Wolgadeutschen, aber auch von Deutschen aus der Zips und von so genannten Schwarzmeerdeutschen sind, soweit ich sehe, so gut wie nie bekannt geworden, ganz zu schweigen von den Deutschen in Übersee, die Klein ausführlich behandelt. Hier ist der Abstand von der „binnendeutschen“ Literatur mehr oder minder unüberbrückbar.

Aufschlussreich sind die Umstände, unter denen Müller-Guttenbrunn vom Wiener Literaten zum donauschwäbischen Autor geworden ist (Klein 1939: 256ff.). Einerseits 
hat ihn der madjarische Nationalismus der Milleniums-Zeit dazu veranlasst ein Buch über die Leistungen der Donauschwaben zusammenzustellen (Deutsche Kulturbilder aus Ungarn, 1896), andererseits hat ihn der an sich wenig profilierte und wohl kurzlebige Akademische Verlag in Wien offenbar aus politischen Gründen 1907 zu dem Roman Götzendämmerung. Ein Kulturbild aus dem heutigen Ungarn angeregt, der, in der Zeit der radikal madjarisierenden Schulgesetze des Grafen Apponyi, eine Auseinandersetzung mit der ungarischen Nationalitätenpolitik ist, welche die Menschen in der österreichischen Reichshälfte zu interessieren begann. Von da war der Weg zu seinen historischen Romanen über die Donauschwaben, die im - deutschnationalen! - Leipziger Staackmann-Verlag recht hohe Auflagen erreichten, nicht mehr weit.

Nicht anders gaben für das Interesse binnendeutscher Verlage an siebenbürgischen Autoren in den 30er Jahren politische Gründe den Ausschlag. Meschendörfers Stadt im Osten ist gewiss kein schlechterer Roman als viele damals in Österreich und im Deutschen Reich entstandene Bücher, aber sie ist auch kein überragendes Werk, wie Klein zu meinen scheint, wenn er schreibt: „Es war Adolf Meschendörfer [...], der mit seinem Roman ,Die Stadt im Osten' 1931 das Eingehen der Siebenbürger in das zeitgenössische deutsche Schrifttum entschied.“ (Klein 1939: 417) In Wahrheit verhält es sich so, dass der zuerst in Hermannstadt erschienene Roman vom rechten Langen Müller Verlag in München übernommen worden ist (und dann in Deutschland recht erfolgreich war), weil die Sprachinseln für das nach rechts rückende deutsche Reich politisch interessant geworden waren.

Das gilt erst recht für die Jahre ab 1933, in denen es zumindest ein Autor aus den auslanddeutschen Sprachgruppen, Karl v. Möller (1876-1943) aus dem Banat, der sich obendrein politisch im Sinn des Nationalsozialismus betätigte, bis in den parteieigenen Eher-Verlag brachte. ${ }^{9}$ Was Klein für einen literarischen Durchbruch gehalten hat, war in Wahrheit politischer Missbrauch - und somit unter geänderten Bedingungen völlig folgenlos, wo nicht schädlich.

Den rein politisch motivierten Zugriff von NS-Verlagen auf Schriftsteller aus den südostdeutschen Minderheiten - neben Meschendörfer auch Wittstock und Zillich - hat Stefan Sienerth (2008c) ausführlich dargestellt. Ich beschränke mich auf eine aussagekräftige, wenn auch wohl zugespitzte Erinnerung von Andreas Birkner (1911-1998), von dem in den frühen 40er Jahren drei Bücher im Stuttgarter Hohenstaufen-Verlag erschienen waren. In einem Gespräch mit Sienerth sagte der Autor zu diesen frühen Veröffentlichungen: „Da hatte ein Stuttgarter Verlag, konjunkturwitternd, die Idee, sich eines weiteren Autors aus Siebenbürgen zu versichern [...]. So kam ich zum Verlag.“ (Sienerth 1997: 141f.) Auf das Wort „konjunkturwitternd“ kommt es an; die Verlage suchten nicht gute Autoren, die eben auch in Siebenbürgen zu finden waren, sondern sie suchten Autoren aus Siebenbürgen, weil die Politik an Autoren mit dieser Herkunft Interesse hatte.

Hans Bergel sieht sehr nüchtern in der literarischen Blüte Siebenbürgens in den dreißiger Jahren eine „Scheinblüte“:

9 Z. B. Karl v. Möller: Die Salpeterer. Ein Freiheitskampf deutscher Bauern. München: Eher 1939. Vorher waren Bücher Möllers im ausgesprochen ,,völkischen“ Wiener Luser Verlag erschienen. 
Denn es kann heute nicht mehr übersehen werden, dass - ungeachtet des Könnens dieser Autoren - im Deutschland der dreißiger und Anfang der vierziger Jahre die aus politischem Kalkül betriebene Förderung auslandsdeutscher Literatur deren Auflagenhöhen erklärt; ohne diese Absicht der offiziösen Kulturpolitik wären Verbreitung und öffentliche Resonanz der Literatur der Südostdeutschen in Deutschland während dieses Zeitabschnitts nicht zustande gekommen. (Bergel 1988: 76)

In der Zeit nach 1945 hatte sich dann alles total verändert. Paul Celan, wohl der wichtigste Autor aus einer der deutschen Sprachinseln, hatte angesichts der überragenden Qualität seiner Gedichte keine Probleme einen Verlag zu finden. Und dennoch: Selbst seine ersten Kontakte zur Wiener Literaturszene und damit der Weg zum ersten Buch wurden durch einen Brief des Czernowitzer bzw. Bukarester Mentors Alfred Margul-Sperber an den Wiener Lyriker und Herausgeber des silberboots, Ernst Schönwiese, erleichtert (Goßens 2001).

Der Ruhm Celans und das Wissen über seine Biografie haben die Entdeckung der gesamten Literatur der Bukowina nach 1970 wo nicht ausgelöst, so doch gefördert (M. Klein 1990: 434). Auffällig ist, dass die Werke der weniger bekannten Czernowitzer in einem auf sie spezialisierten Verlag erschienen sind, dem Rimbaud Verlag in Aachen, der allerdings erst in den 90er Jahren des vergangenen Jahrhunderts aktiv wurde. Rose Ausländer, die seit 1956 im Westen (zuerst in Wien, vorher schon in den USA) lebte, trat etwas früher in die Literaturszene ein.

Sonst sind mit wenigen Ausnahmen ${ }^{10}$ nach 1945 in der Bundesrepublik, in der Deutschen Demokratischen Republik und in Österreich kaum Werke von Autoren aus den Minderheitengebieten erschienen. Ein Sonderfall ist der Verlag des Südostdeutschen Kulturwerks, der gelegentlich sogar ältere Texte von deutschen Autoren und Autorinnen aus dem Südosten druckte - aber im Wesentlichen für Leser und Leserinnen aus dem Südosten, denn wirklich präsent im Buchhandel war der sehr verdienstliche Verlag nicht.

Im Zusammenhang mit diesem Verlag noch eine Anmerkung zur wissenschaftlichen Rezeption der Literatur aus den ostmitteleuropäischen Sprachinseln, die zumeist in Publikationen dieses Verlags und in seiner Zeitschrift erfolgt ist. Die meisten Germanisten und Germanistinnen, die sich mit der Literatur aus Siebenbürgen, dem Banat und der Bukowina beschäftigt haben, stammen - mit Ausnahme derer, die über Paul Celan, Herta Müller und einige wenige andere forschen - selbst aus diesen Regionen; insofern hat die Forschung über diese Teile der deutschen Literatur etwas Insiderisches. ${ }^{11}$ Karl Kurt Kleins große Literaturgeschichte des Deutschtums im Ausland ist der beste Beweis dafür - denn der Siebenbürger Klein war ein sehr bewusster Auslanddeutscher und betrachtete die von ihm vorgestellte Literatur sehr wohl aus der Innensicht.

10 U. a. Paul Schuster: Fünf Liter Zuika. Graz: Stiasny 1968 (7 Jahre nach der Veröffentlichung in Rumänien) und zwei Romane von Cisek bei Suhrkamp. Cisek lebte und schrieb zwar in Rumänien, ist aber keiner der historischen Sprachinseln zuzuordnen. Bücher von ihm erschienen vor 1945 ausschließlich in Deutschland; erst nach dem Zweiten Weltkrieg hat er auch in Rumänien publiziert.

11 Eine der wenigen Ausnahmen ist Dieter Kessler, eine andere Alexander Ritter, der sich eher allgemein mit auslanddeutscher Literatur befasst hat. 
Schließen möchte ich mit einem konkreten Beispiel, das zeigt, dass manche Strukturen der Rezeption von Autoren und Autorinnen aus den deutschen Minderheiten sogar die große Einwanderungswelle überlebt haben.

Der Roman Amei und Mari oder Nacherzähltes Leben mit dem vieldeutigen Untertitel Ein Heimatroman von dem Banater Autor Johann Lippet ist 2015 im POP Verlag in Ludwigsburg erschienen. Schon der Verlag ist bemerkenswert, denn Veröffentlichungen von rumäniendeutschen Autoren und Autorinnen sind eindeutig ein Schwerpunkt seiner Programme; man kann durchaus vermuten, dass der Verlag sich auch an ein aus Rumänien stammendes Publikum wendet - wie eben vor 1939 die kleinen Verlage in den Minderheitengebieten, zugespitzt könnte man ihn als rumänisch/rumäniendeutschen Exilverlag bezeichnen.

Auch das beeindruckende Buch selbst wendet sich an Leser und Leserinnen, die mit der jüngsten Geschichte der Donauschwaben halbwegs vertraut sind. Zwar erzählt Lippet auch historische Fakten wie den Frontenwechsel Rumäniens 1944 oder die Enteignung der deutschen Bauern, die aus dem Banat stammenden Menschen bekannt sein müssen, aber im Vordergrund des tieftraurigen Buchs steht das fast nostalgisch heraufbeschworene donauschwäbische Dorf; jene Informationen mögen vor allem für Leser und Leserinnen bestimmt sein, die wenig vom Schicksal der Deutschen in Rumänien wissen und die der Roman dennoch ansprechen will. Insofern wendet er sich an ein breiteres Publikum als die ältere Literatur aus den Minderheitsgebieten; Lippet will, um das Urteil über Michael Albert abzuwandeln, nicht nur ein Banater Dichter sein, aber sein Buch soll trotzdem auch gemeinschaftsstiftend für die Landsleute sein - eine Funktion, die die Literatur aus dem Kreis der Sprachminderheiten bewahrt. ${ }^{12}$

\section{Primärliteratur}

HODJAK, Franz (1988) Sehnsucht nach Feigenschnaps. Ausgewählte Gedichte. Hrsg. von Wulf Kirsten. Berlin/Weimar: Aufbau.

LIPPET, Johann (2015) Amei und Mari oder Nacherzähltes Leben. Ein Heimatroman. Ludwigsburg: POP.

MARGUL-SPERBER, Alfred (1973) Verzaubertes Wort. Berlin: Verlag der Nation.

MESCHENDÖRFER, Adolf (1931) Die Stadt im Osten. Roman. Hermannstadt: Krafft \& Drotleff.

SCHLATTNER, Eginald (2012) Mein Nachbar, der König. Verlassene Geschichten. Hrsg. von Michaela Nowotnick. Hermannstadt/Bonn: Schiller.

SCHLATTNER, Eginald (2012) Odem. Kritische Edition. Hrsg. von Michaela Nowotnick. Hermannstadt/Bonn: Schiller.

SCHLATTNER, Eginald (2018) Wasserzeichen. Roman. Ludwigsburg: POP.

\section{Sekundärliteratur}

BERGEL, Hans (1988) Literaturgeschichte der Deutschen in Siebenbürgen. Ein Überblick. 2. Aufl. Thaur: Wort und Welt.

12 Was auf Amei und Mari zutrifft, gilt nicht für Lippets Lyrik, zumindest nicht für große Teile davon. 
GADEANU, Sorin (2011) „,Mit den Hinterbeinchen kleben, mit den Vorderbeinen schreiben': Zur ,kleinen Literatur' des deutschsprachigen Banats." In: Ch. Fackelmann (Hrsg.), Literatur - Geschichte - Österreich. Thematische Festschrift für Herbert Zeman. Wien: LIT, 295-308.

GOSSENS, Peter (2001) „Ernst Schönwiese, Otto Basil und der Plan.“ In: P. Goßens/M. G. Patka (Hrsg.): ,Displaced'. Paul Celan in Wien 1947-48 [Zur Ausstellung im Jüdischen Museum Wien]. Frankfurt: Suhrkamp, 53-62.

JACHIMOWICZ, Aneta (2017) Der historische Roman der Ersten Republik in ideologiekritischer Sicht. Würzburg: Königshausen \& Neumann.

KLEIN, Karl Kurt (1939) Literaturgeschichte des Deutschtums im Ausland. Leipzig: Bibliographisches Institut. Hildesheim: Olms 1979.

KLEIN, Michael (1990) „Zur Präsenz der Bukowina im westlichen deutschsprachigen Literaturbetrieb.“ In: D. Goltschnigg/A. Schwob (Hrsg.). Die Bukowina. Studien zu einer versunkenen Literaturlandschaft. Tübingen: Francke, 429-442.

MILLNER, Alexandra (2016) „Schmerz, Erbarmen und Lebensnerv. Marie Eugenie delle Grazies Drama ,Schlagende Wetter' (1898) - über den Naturalismus hinaus gelesen." In: R. Innerhofer/D. Strigl (Hrsg.), Sonderweg in Schwarzgelb? Auf der Suche nach einem österreichischen Naturalismus in der Literatur. Innsbruck: Studienverlag, 171-188.

NAGL, Johann Willibald/Jakob ZEIDLER/Eduard CASTLE (Hrsg.) (1899-1937) Deutsch-Österreichische Literaturgeschichte. 4 Bände. Wien: Fromme.

SCHEICHL, Sigurd Paul (1992) „Südostdeutscher Autor - binnendeutscher Verlag binnendeutscher Leser." In: A. Schwob (Hrsg.), Die deutsche Literaturgeschichte Ostmittel- und Südosteuropas von der Mitte des 19. Jahrhunderts bis heute. Forschungsschwerpunkte und Defizite. München: Südostdeutsches Kulturwerk, 43-57.

SCHEICHL, Sigurd Paul (1993) „Der Austritt aus der Regionalliteratur.“ In: A. Schwob/B. Tontsch (Hrsg.), Die siebenbürgisch-deutsche Literatur als Beispiel einer Regionalliteratur. Köln: Böhlau, 33-49.

SCHEICHL, Sigurd Paul (2019; zuerst 2009) „,Wie an den Lagerfeuern der Karawansereien'. Eginald Schlattner - ein exotisches Kuriosum? Zur Schlattner-Rezeption in den deutschsprachigen Ländern." MATRIX. Zeitschrift für Literatur und Kunst $1 / 2019$ (55), 27-49.

SIENERTH, Stefan (Hrsg.) (1997) „Daß ich in diesen Raum hineingeboren wurde ..." Gespräche mit deutschen Schriftstellern aus Südosteuropa. München: Südostdeutsches Kulturwerk.

SIENERTH, Stefan (2008a) Studien und Aufsätze zur Geschichte der deutschen Literatur und Sprachwissenschaft in Südosteuropa. 2 Bände. München: IKGS.

SIENERTH, Stefan (2008b) „Der siebenbürgisch-deutsche Autor im Dilemma der Entscheidung zwischen dem heimischen und dem europäischen Adressatenkreis." [1993]. In: Sienerth (2008a), Band 2, 37-55.

SIENERTH, Stefan (2008c) „Adolf Meschendörfer und Heinrich Zillich im Literaturbetrieb des ,Dritten Reiches'“. In: Sienerth (2008a), Band 2, 189-226. 


\section{Zusammenfassung \\ DAS ,ZENTRUM“ UND DIE LITERATUR VON DEN „RÄNDERN“. DIE REZEPTION DER LITERATUR DER DEUTSCHEN MINDERHEITEN IN DEUTSCHLAND UND ÖSTERREICH}

Wenige Autoren und Autorinnen aus den deutschen Sprachinseln Österreich-Ungarns, Rumäniens, Russlands usw. fanden Leser und Leserinnen in Deutschland und Österreich, mit Ausnahme jener, die aus ihrer Heimat dahin übersiedelt waren. Gründe dafür sind einerseits die Orientierung der Bücher am Publikum in den Sprachinseln, andererseits das Desinteresse der Verlage an den manchmal etwas altmodischen Werken. Allein die Nationalsozialisten förderten aus politischen Gründen die Drucklegung von Büchern aus den betreffenden Regionen. Nach 1945 hat sich das durch die Übersiedlung vieler Autoren und Autorinnen aus den Sprachinseln in die Bundesrepublik geändert.

Schlüsselwörter: auslanddeutsche Literatur, deutsche Sprachinseln, Rezeption der Literatur

Abstract

THE “CENTER” AND THE LITERATURE FROM THE “PERIPHERIES”. THE RECEPTION OF THE LITERATURE OF GERMAN MINORITIES IN GERMANY AND AUSTRIA

Very few authors from ethnic German minorities in for example Austria-Hungary, Rumania and Russia found readers in German-speaking countries, that is primarily Germany and Austria, with the exception of writers who had moved to cities such as Vienna and Berlin. One reason is that these authors targeted a local audience, another is that publishers were not interested in their work, which often was rather traditionalist. Only the national socialists had books from regions such as Bukovina and Transylvania published in Germany for political reasons. This has changed after 1945, when many important authors left the minority regions and moved to Germany.

Keywords: literature of the German diaspora, German language islands, reception of literature

\section{Povzetek}

„SREDIŠČE” IN KNJIŽEVNOST „Z OBROBIJ”.

\section{RECEPCIJA KNJIŽEVNOSTI NEMŠKIH MANJŠIN V NEMČIJI IN AVSTRIJI}

Le redke avtorice in avtorji iz nemških jezikovnih otokov Avstro-Ogrske, Romunije, Rusije itd. so v nemško govorečih deželah, predvsem Nemčiji in Avstriji, našli pot do bralstva; izjema so bili le tisti avtorji, ki so se iz svoje domovine izselili na Dunaj, v 
Berlin itd. Po eni strani je razlog za tako skromno recepcijo mogoče najti v usmerjenosti knjižnih del teh avtorjev, ki so bila naslovljena predvsem na bralstvo $\mathrm{v}$ jezikovnih otokih, po drugi strani pa razlog tiči tudi v nezinteresiranosti založb za marsikdaj tradicionalno obvarvana dela. Iz političnih razlogov so bili zgolj nacionalsocialisti tisti, ki so spodbujali tiskanje knjig iz omenjenih regij. Recepcija pa se je spremenila po letu 1945, ko so se mnoge avtorice in avtorji iz jezikovnih otokov preselili v Zvezno republiko Nemčijo.

Ključne besede: književnost nemške diaspore, nemški jezikovni otoki, recepcija književnosti 